\title{
Optical characteristics of photo-curable methacryl-oligosiloxane nano hybrid thick films
}

\author{
Young-Joo Eo, Jeong Hwan Kim, Ji Hoon Ko, and Byeong-Soo Bae ${ }^{\text {a) }}$ \\ Laboratory of Optical Materials and Coating (LOMC), Department of Materials Science \\ and Engineering, Korea Advanced Institute of Science and Technology (KAIST), \\ Daejeon 305-701, Republic of Korea
}

(Received 17 August 2004; accepted 28 October 2004)

\begin{abstract}
In this paper, we report the fabrication the methacryl-oligosiloxane nano hybrid films from 3-(trimethoxysilyl)propyl methacrylate (MPTS) and diphenylsilanediol (DPSD), and the investigation of the tuneability of the optical characteristics of the films through the compositional change. The viscosity of the methacryl-oligosiloxane nano hybrid resin was altered at $10^{2}$ intervals by the compositional modification without any drastic changes in the reaction parameters, and thickness-controlled (from 11 to $150 \mu \mathrm{m}$ ) and uniformly coated (less than $1 \mathrm{~nm}$ root-mean-square roughness) films were obtained through a single coating step. The refractive indices were tunable from 1.506 to 1.543 , depending on composition. On the other hand, the thermo-optic coefficients remained constant $\left(-2 \times 10^{-4} /{ }^{\circ} \mathrm{C}\right)$, independent of composition. Also, we demonstrated a thick $(170 \mu \mathrm{m})$ photo pattern with a high aspect ratio (3:1).

Methacryl-oligosiloxane nano hybrid materials can be promising candidates for the optical applications due to easy and wide tuneability of their optical parameters.
\end{abstract}

\section{INTRODUCTION}

Planar optical devices have been of much interest in integrated planar lightwave circuit applications. Much effort has been exerted to develop planar optical devices using optically transparent materials such as silica and polymers. ${ }^{1,2}$ They were produced using various methods, such as the physical vapor deposition (PVD), chemical vapor deposition (CVD), and sol-gel processes. Among these, the sol-gel process has been considered the most practical in fabricating the planar optical devices because optically homogeneous coatings can easily be obtained at a low cost. ${ }^{3,4}$

In recent years, sol-gel-derived organic-inorganic hybrid materials (HYBRIMERs) were developed and studied for optical applications because of their excellent optical properties and relatively high thermal stabilities in comparison to general optical polymers. ${ }^{5,6}$ They also possess some very fascinating features, such as their photo-curablility and their easily tunable optical characteristics. ${ }^{7-11}$

A photo-curable HYBRIMER can be synthesized through the introduction of photo-active precursors, which usually contain $\mathrm{C}=\mathrm{C}$ bonds. One of these photocurable HYBRIMERs is the methacryl-oligosiloxane

\footnotetext{
a) Address all correspondence to this author.

e-mail: bsbae@kaist.ac.kr

DOI: $10.1557 / J M R .2005 .0045$
}

nano hybrid material (methacryl HYBRIMER) synthesized from 3-(trimethoxysilyl)propyl methacrylate (MPTS) and diphenylsilanediol (DPSD). MPTS has a photo-polymerizable methacryl group. DPSD contains silanol groups, which can react with the methoxy groups in MPTS. Since methanol, a byproduct of the condensation reaction between the precursors, can easily be removed through vacuum heating, a photo-curable solventless methacryl-oligosiloxane nano hybrid resin can be synthesized. The thick and crack-free methacryl HYBRIMER coating films can be obtained via a photopolymerization reaction of methacryl groups in the resin because the stress on the films induced by the evaporation of the volatile solvent was minimized during the curing step. The schematic reactions of the fabrication of methacryl HYBRIMER films are illustrated in Fig. 1. This methacryl HYBRIMER is a promising candidate for use in photonic devices, since it exhibits low optical absorption caused by few residual silanol contents, good adhesion behavior upon various substrates, high thermal stability, and photo-patternability. ${ }^{12-16}$

We have already reported that the size and shape of oligosiloxane in the resin were altered by the composition ratio of the precursors, MPTS and DPSD, but without any drastic changes in the reaction parameters. ${ }^{17}$ The molecular structural change of the resin directly influences rheological and polymerization behavior. Moreover, the optical properties of the methacryl HYBRIMER can be also altered through compositional modification. 


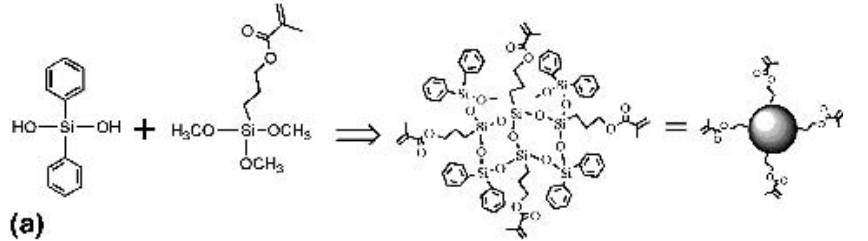

(a)
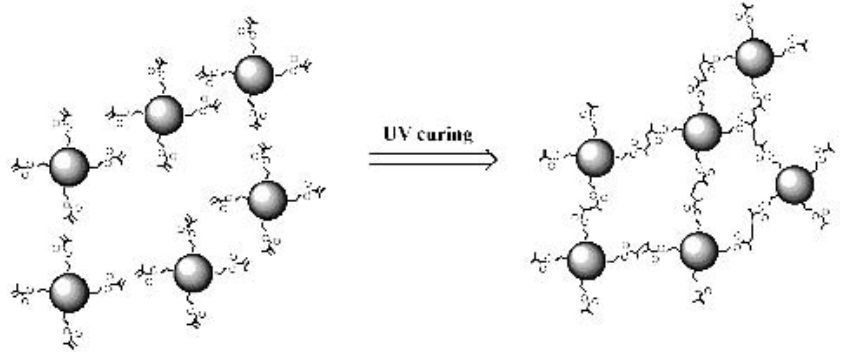

(b)

FIG. 1. Schematic illustration of (a) the synthesis of the methacryloligosiloxane nano hybrid resin via a condensation reaction between the MPTS and the DPSD, and (b) the fabrication of the methacryloligosiloxane nano hybrid materials (methacryl HYBRIMER) through the photo-curing.

The most important aspects in the fabrication of the photonic devices are the precise control of the dimensions of the device structure and the optical properties of the materials. ${ }^{1}$ In this respect, to make the methacryl HYBRIMER fit the requirements of the various optical applications precisely, it is important to study the effects of the compositional modification.

In this study, a thick methacryl-HYBRIMER film was fabircated from DPSD and MPTS. The effects of compositional modification on the rheological and polymerization behaviors and its optical properties, such as the refractive index and the thermo-optic coefficient, were studied. Finally, a photo-pattern of thick methacryl HYBRIMER films with high aspect ratios was also demonstrated.

\section{EXPERIMENT}

\section{A. Fabrication of methacryl HYBRIMER films}

The precursor molecules, used without further purification, were 3-(trimethoxysilyl)propyl methacrylate (MPTS, Aldrich, Milwaukee, WI) and diphenylsilanediol (DPSD, TCI, Tokyo, Japan). Barium hydroxide monohydrate $\left[\mathrm{Ba}(\mathrm{OH})_{2} \cdot \mathrm{H}_{2} \mathrm{O}\right.$, Aldrich] was also used as a catalyst to promote the condensation reaction between the two precursors. MPTS and $\mathrm{Ba}(\mathrm{OH})_{2} \cdot \mathrm{H}_{2} \mathrm{O}$ were mixed at $80{ }^{\circ} \mathrm{C}$, and DPSD was added continuously to the mixture for $2 \mathrm{~h}$ to prevent phase separation and self-condensation of DPSD. The amount of $\mathrm{Ba}(\mathrm{OH})_{2} \cdot \mathrm{H}_{2} \mathrm{O}$ was fixed at $0.1 \mathrm{~mol} \%$ of the total silane compound while that of DPSD was changed from 33 to $55 \mathrm{~mol} \%$ of the total silane compound. The solution was kept at $80{ }^{\circ} \mathrm{C}$ for another $2 \mathrm{~h}$ to progress the reaction. After that, methanol, a byproduct of the condensation reaction, was removed by vacuum heating. The solution was allowed to cool at room temperature and was later filtered using a $0.45-\mu \mathrm{m}$ Teflon filter, to remove $\mathrm{Ba}(\mathrm{OH})_{2} \cdot \mathrm{H}_{2} \mathrm{O}$. This left a clear solution of solvent-less methacryl-oligosiloxane nano hybrid resin.

For the photo-polymerization of the methacryl groups in the resin, 2,2-dimethoxy-2-phenyl-acetophenone (BDK, Aldrich) and 2-benzyl-2-N,N-dimethylamino-1(4-morpholinophenyl)butanone (IRG 369, Aldrich) were added as a photo initiator before the coating process. The photo initiator had a concentration level of $1 \mathrm{~mol} \%$ of the total polymerizable methacryl group. The prepared photo-sensitive resins were spin-coated onto the silicon wafers at various rpms for $30 \mathrm{~s}$ The resultant films were illuminated using an ultraviolet (UV) lamp (500W Hg Lamp, $\sim 365 \mathrm{~nm}$, Oriel 97453) in an $\mathrm{N}_{2}$ atmosphere. The HYBRIMER films were thermally treated for better curing for $4 \mathrm{~h}$ at $150{ }^{\circ} \mathrm{Cl}$ after the photo-polymerization.

\section{B. Characterization}

The near infrared (NIR) absorption spectrum of the resin was obtained using an ultraviolet-visible-near infrared (UV/VIS/NIR) spectrophotometer (Shimadzu, Kyoto, Japan, UV-3101PC). The viscosity of the resin was measured at $30{ }^{\circ} \mathrm{C}$ using a rheometer (BROOKFIELD, DV-III+). The film-thickness measurement was carried out using a scanning electron microscope (SEM; Phillips 535M). The surface morphology of the film was observed using a atomic force microscopy (AFM; Autoprobe 5M, Park Scientific Instruments, Woodbury, NY). The structural evolutions of the films were examined through Fourier transform infrared (FTIR) spectroscopy (JASCO, FT-IR 460plus). All measurements were performed at a $4000-500 \mathrm{~cm}^{-1}$ range with a $4 \mathrm{~cm}^{-1}$ resolution. The refractive indices of the resins were determined using the Abbe refractometer (Bellingham Stanley Ltd. 60/ED), at a 589.6-nm wavelength. The refractive index of the coating film was obtained through a prism coupling method at a $1550-\mathrm{nm}$ wavelength. The prism coupler (Pennington, Metricon 2010), equipped with an auto-controlled hot stage, was used to measure the temperature dependence of the refractive index $(\mathrm{d} n / \mathrm{d} T)$ in the coating films. ${ }^{7,18}$ The refractive index was measured at a temperature range of $30-90{ }^{\circ} \mathrm{C}$.

\section{RESULTS AND DISCUSSIONS}

\section{A. Fabrication of thick films with low optical absorption}

An important issue of materials for optical application is the NIR absorption behavior, particularly at 1310 and $1550 \mathrm{~nm} .{ }^{1,13}$ The methacryl-oligosiloxane nano hybrid resin can be synthesized through a condensation reaction 
between the silanol group of DPSD and the methoxy group of MPTS. This synthesis is carried out without the addition of water, which can reduce the residual silanol contents. ${ }^{13}$ Methanol, a byproduct of the condensation reaction between the precursors, can easily be removed through vacuum heating, as previously mentioned. Thus, the amount of $\mathrm{CH}$ and $\mathrm{OH}$ groups in the resin can be decreased and, consequently, the NIR absorption of the resin at 1310 and $1550 \mathrm{~nm}$ can be minized. Figure 2 presents the NIR absorption spectra of the resin with different compositions. As shown in Fig. 2, the resin shows less than $1 \mathrm{~dB} / \mathrm{cm}$ of optical absorption at telecom wavelength (at 850,1310 , and $1550 \mathrm{~nm}$ ), this value is lower than that of optical poly-methyl methacrylate (PMMA) whose absorption at $1550 \mathrm{~nm}$ is around $1.5 \mathrm{~dB} / \mathrm{cm}^{13}$

The degree of the condensation reaction of the resin during the synthesis can be controlled by the composition ratio of the precursors, DPSD and MPTS. ${ }^{17}$ This directly affects the size and shape of oligosiloxane in the resin. Thus, the rheological behavior of the resin can be changed through compositional modification. Figure 3(a) shows the viscosity of the resin as a function of composition. The viscosity increases from 30 to $1800 \mathrm{cps}$ with DPSD contents. This was caused by the further condensation reactions and, consequently, by the increase of the molecular weight of the resin. And also, it was found that when the DPSD content was $50 \mathrm{~mol} \%$ or less, the oligosiloxanes in the resins were mostly linear in shape. ${ }^{17}$ When the DPSD content was more than $50 \mathrm{~mol} \%$, on the other hand, the oligosiloxane in the resin branched out because of the stoichiometry between the silanol groups of DPSD and the methoxy groups of MPTS. ${ }^{17}$ The abrupt increase in the viscosity of the resins at $55 \mathrm{~mol} \%$ of DPSD contents can, therefore, be explained by the formation of the branched oligosiloxane. ${ }^{19}$

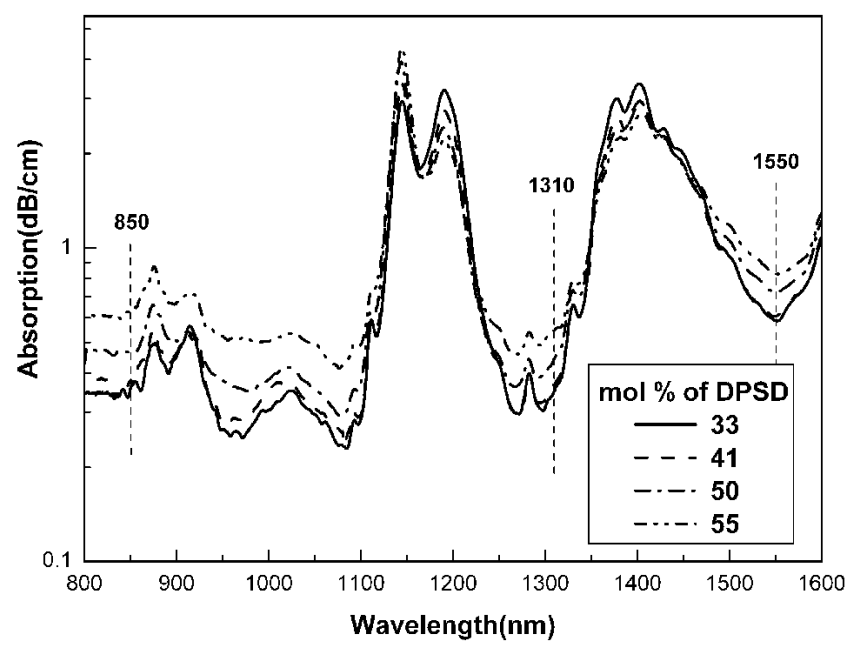

FIG. 2. NIR absorption spectra of the methacryl-oligosiloxane nano hybrid resin with different compositions.
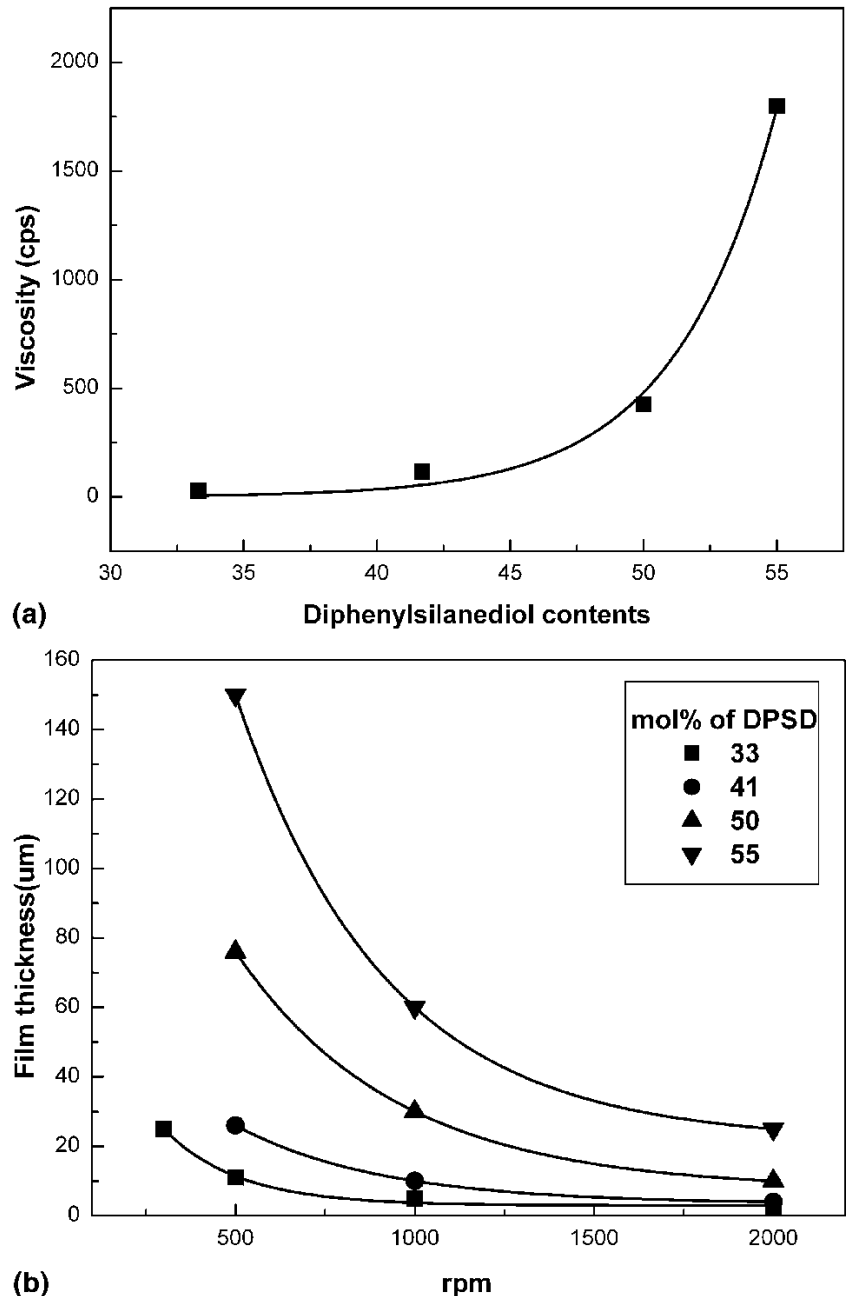

FIG. 3. (a) Viscosity of the methacryl-oligosiloxane nano hybrid resin as a function of composition, and (b) methacryl HYBRIMER film thickness with different compositions as a function of rpm.

The spin-coated methacryl HYBRIMER film thickness can be controlled by its composition and spincoating speed (rpm). Figure 3(b) shows the thickness of the spin-coated film with different compositions as a function of rpm. It was found that all of the films had no cracks, although their thickness levels rose to $150 \mu \mathrm{m}$. The film thicknesses increased from 11 to $150 \mu \mathrm{m}$, in response to the DPSD contents, at a constant 500-rpm condition caused by the viscosity change. They were also widely tunable through the modification of the spincoating speeds, especially from 30 to $150 \mu \mathrm{m}$ in the case of $55 \mathrm{~mol} \%$ of DPSD contents.

Figure 4 shows the cross-sectional SEM images of the films in different compositions, at constant $500 \mathrm{rpm}$ conditions. The surfaces of the films of all the compositions were flat and uniform. Their root-mean-square (rms) roughnesses, measured by the AFM, were all less than $1 \mathrm{~nm}$. The films also exhibited good wetting and adhesion behavior on the substrate. This thickness-controlled 


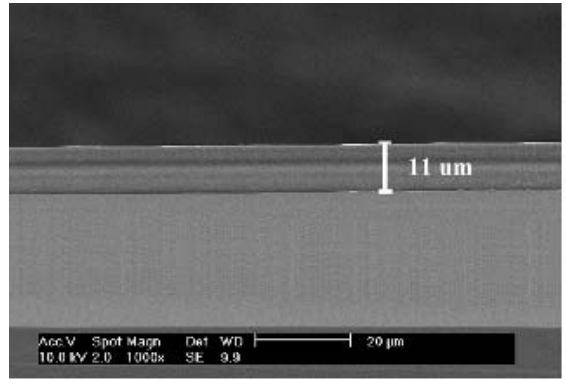

(a)

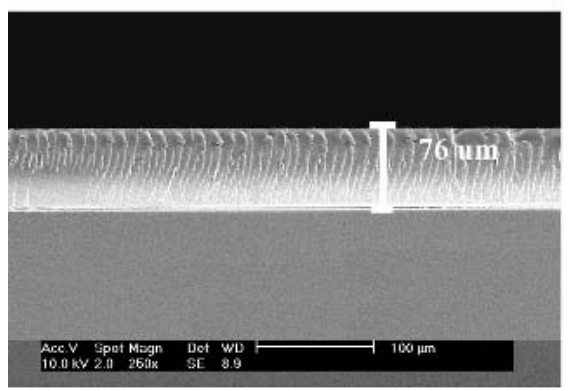

(c)

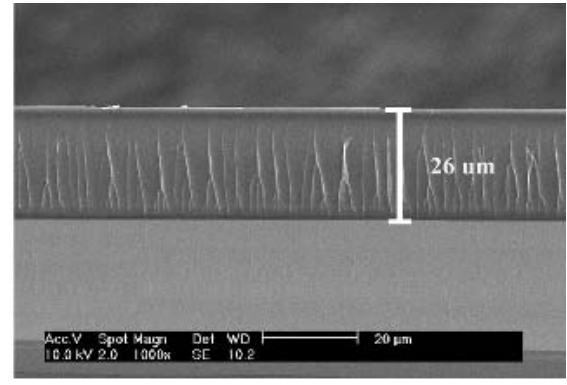

(b)

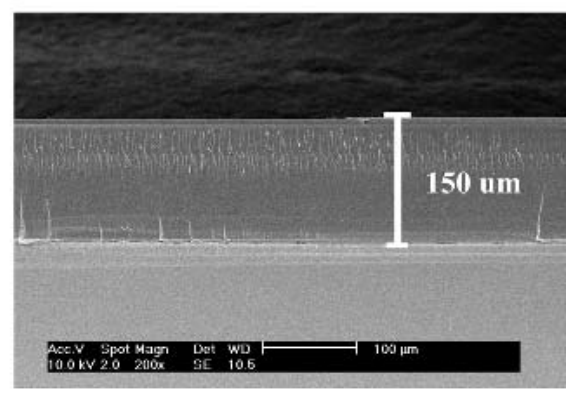

(d)

FIG. 4. Cross-sectional SEM images of methacryl HYRIMER films with (a) $33 \mathrm{~mol} \%$, (b) $41 \mathrm{~mol} \%$, (c) 50 mol $\%$, and (d) $55 \mathrm{~mol} \%$ DPSD contents, obtained from single spin coating at $500 \mathrm{rpm}$.

and flaw-less methacryl HYBRIMER films can, therefore, even be used in multilayer optical applications.

\section{B. Photo-curing behavior}

Photo-curing through the polymerization of the methacryl groups in the resin can be confirmed using FTIR spectroscopy. Figure 5 shows the FTIR spectra of the films doped with BDK as the photo-initiator. The peak assignments of the FTIR spectra have been recorded in previous works. ${ }^{12}$ In the FTIR spectra, the 1720 and 1640 $\mathrm{cm}^{-1}$ peaks represent the carbonyl $(\mathrm{C}=\mathrm{O})$ group stretching mode and the vinyl $(\mathrm{C}=\mathrm{C})$ group stretching mode,
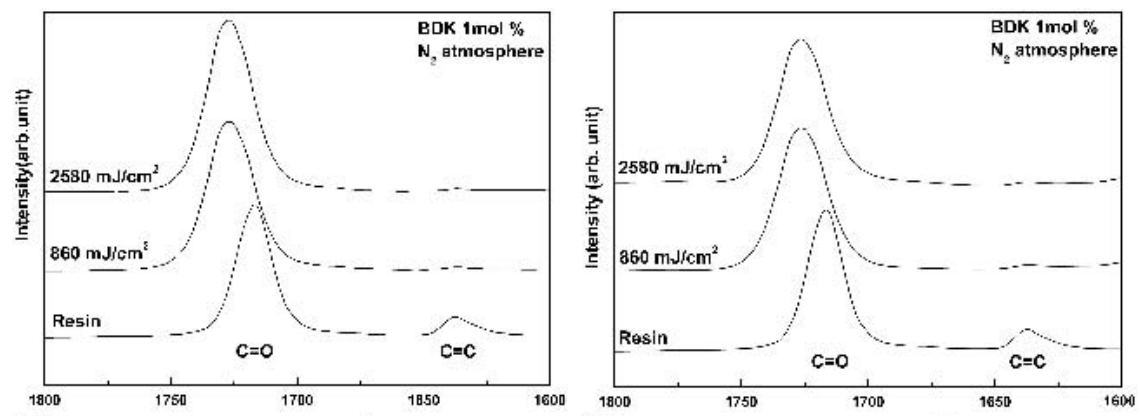

(a)

wavenumber $\left(\mathrm{cm}^{1}\right)$

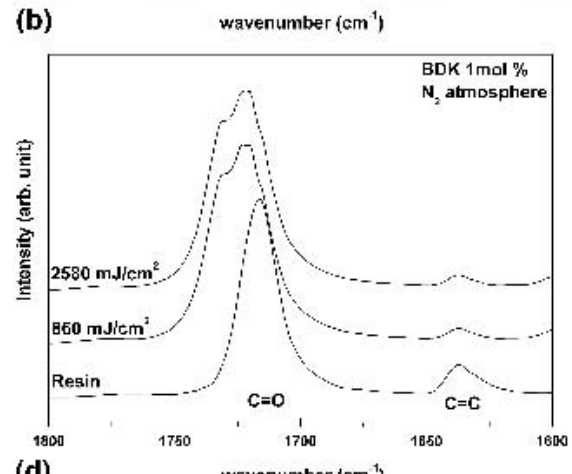

(c)

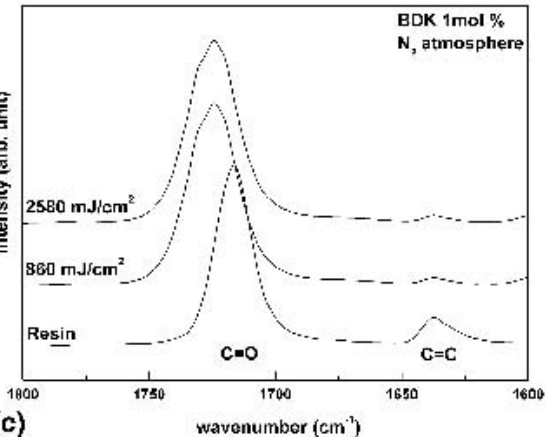

(d)

wavenumber $\left.\left(\mathrm{cm}^{-1}\right)\right\}$

FIG. 5. FTIR spectra of methacryl HYBRIMER films with (a) $33 \mathrm{~mol} \%$, (b) $41 \mathrm{~mol} \%$, (c) $50 \mathrm{~mol} \%$, (d) 55 mol\% DPSD contents, as a function of UV doses. 
respectively. The intensity of the peak from the $\mathrm{C}=\mathrm{C}$ group stretching mode decreased with UV irradiation. This indicates that the photo-polymerization of the methacryl groups has proceeded. The $\mathrm{C}=\mathrm{O}$ peak shifts to higher wave numbers as the photo-polymerization continues, showing that the $\mathrm{C}=\mathrm{O}$ group does not conjugate with the $\mathrm{C}=\mathrm{C}$ group. The decreases in the $\mathrm{C}=\mathrm{C}$ peak intensity levels in all the compositions were saturated only at UV doses of $860 \mathrm{~mJ} / \mathrm{cm}^{2}$. This indicates that the photo-polymerization efficiency of the methacryl HYBRIMER is very high compared to other nano hybrid materials. ${ }^{7,9-11}$ It was also found that the photopolymerization behavior of methacryl HYBRIMER films, doped with IRG 369 as photo-initiators, was very similar to that of BDK from the FTIR spectroscopy. Based on the FTIR spectra shown in Fig. 5, the conversion degree of the $\mathrm{C}=\mathrm{C}$ group may be calculated using Eq. $(1)^{12}$

$$
\begin{aligned}
& \text { Conversion degree }(\%)= \\
& \qquad\left(1-\frac{\left(\frac{A_{\mathrm{C}=\mathrm{C}}}{A_{\mathrm{C}=\mathrm{O}}}\right)_{\text {after UV }}}{\left(\frac{A_{\mathrm{C}=\mathrm{C}}}{A_{\mathrm{C}=\mathrm{O}}}\right)_{\text {Resin }}}\right) \times 100,
\end{aligned}
$$

where $A_{C=C}$ and $A_{C=O}$ represent the integrated peak intensities of the $\mathrm{C}=\mathrm{C}$ and $\mathrm{C}=\mathrm{O}$ groups, respectively.

Figure 6 shows the conversion degrees of the methacryl HYBRIMER films as functions of composition. Based on this, it can be deduced that, despite the small UV doses, organic networks can be sufficiently formed, regardless of the photo-initiators. The conversion degrees of the films decreased with the increase in the DPSD contents. As mentioned previously, the viscosity of the

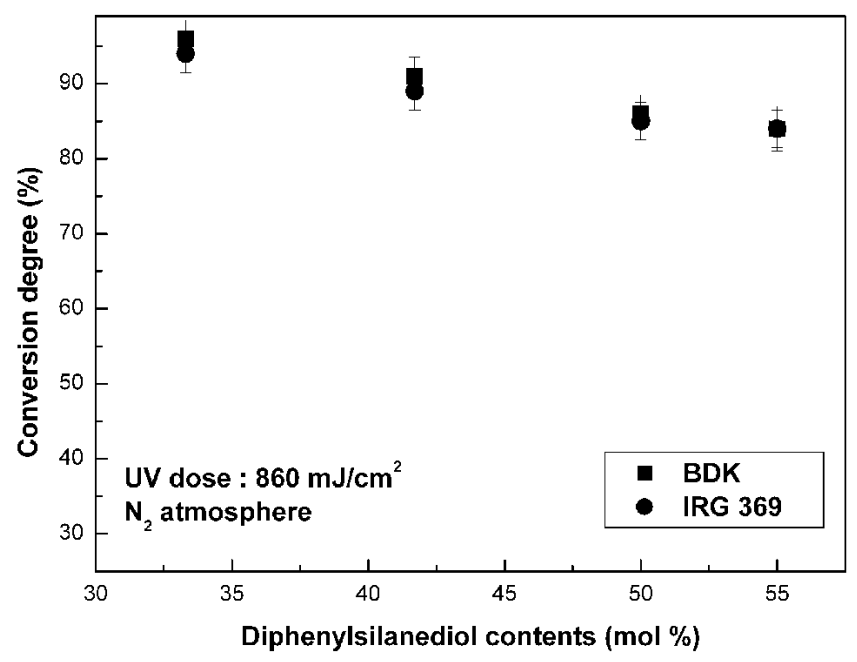

FIG. 6. Conversion degree of methacryl group in methacryl HYBRIMER films with different photo-initiators as a function of composition. resin and the amount of phenyl groups in the resin increase with the DPSD contents. The polymerization reaction between methacryl groups can, therefore, be sterically inhibited, resulting in a decrease in the conversion degree of the films.

\section{Refractive index}

To study the effects of composition on optical parameters, the refractive indices of resin and film were measured. Figure 7 presents the refractive index of the resin as functions of compositions and the films of the corresponding composition. The refractive indices of the resins increase from 1.494 to 1.545 with DPSD contents at a 589.6-nm wavelength. Those of the films, on the other hand, were changed from 1.506 to 1.543 at a $1550-\mathrm{nm}$ wavelength.

A refractive index change $\Delta n$ is represented by the differential form of the Lorentz-Lorenz equation ${ }^{11}$

$$
\Delta n=\frac{\left(n^{2}-1\right)\left(n^{2}+2\right)}{6 n}\left(\frac{\Delta \alpha}{\alpha}+\frac{\Delta \rho}{\rho}\right)
$$

where $\alpha$ and $\rho$ represent polarizability and density, respectively.

Based on Eq. (2), it can be expected that the refractive index will increase with polarizability and densification. In case of resin, it was found that the refractive index change, between 33 and 55 mol\% of DPSD contents, by the densification was 0.0033 , which was determined by measuring the densities of the resins. This value is negligible compared to the total refractive index change of resin. Therefore, due to a higher electronic polarizability of the phenyl-containing DPSD precursor compared to that of the MPTS precursor, the refractive index of the resin linearly increased with the DPSD contents.

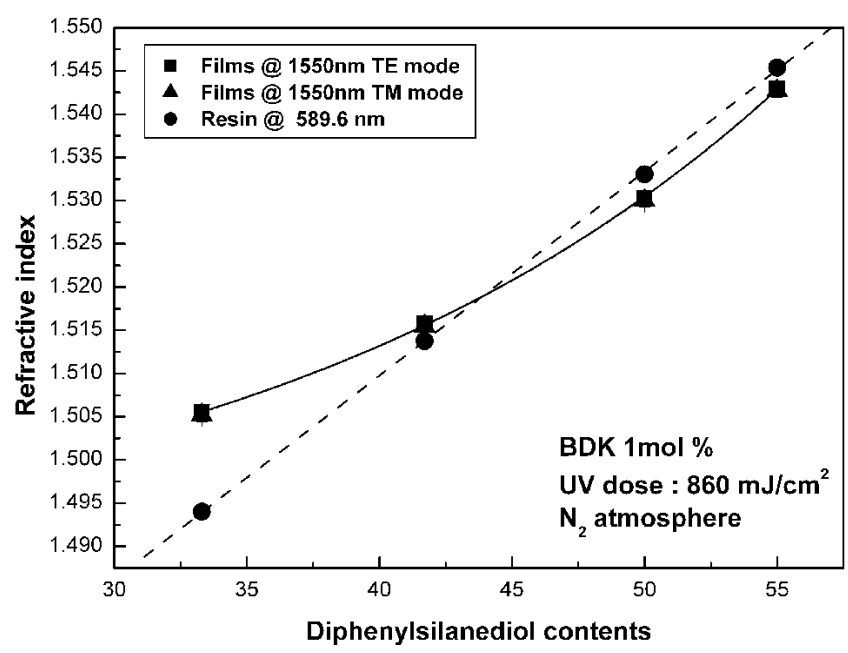

FIG. 7. Refractive index changes in the methacryl-oligosiloxane nano hybrid resin and the methacryl HYRIMER films, as a function composition. 
However, the refractive index change in the coating films is somewhat different. As shown in Fig. 7, the refractive index of the film increases nonlinearly with DPSD contents. This can be explained by the densification of the films. During the fabrication of the films through photo-curing, the methacryl groups of the resin were polymerized. Their conversion degrees increased with the decrease in DPSD contents, as shown in Fig. 6. More methacryl groups and higher conversion degrees can make the films more densified during photo-curing. It has been reported that the photo-polymerization of the methacryl groups can increase the refractive index of the film through densification. ${ }^{7,9-11}$ The refractive index change of the film with low DPSD contents, therefore, becomes larger than those with high DPSD contents during photo-polymerization. This densification-induced refractive index change seems not to be negligible, in case of the coating films.

The films also exhibited a low birefringence $\left(3 \times 10^{-4}\right)$, independent of composition, at a $1550-\mathrm{nm}$ wavelength.

\section{Thermo-optic coefficient}

The thermo-optic effect is defined as the temperature dependence of the refractive index of a material. The thermo-optic characteristic is of considerable importance to the design of optical devices, such as optical switches and athermal devices. ${ }^{1}$ Figure 8 illustrates the refractive index change of the films, at different compositions, as a function of temperature. In all the compositions, the refractive indices of the films decreased linearly with temperature. In the linear plot of the refractive index change against temperature, therefore, the thermo-optic coefficient $(\mathrm{d} n / \mathrm{d} T)$ of the film can be calculated from its gradients. ${ }^{7,18}$ As shown in Fig. 8, their $\mathrm{d} n / \mathrm{d} T$ s are $-2 \times 10^{-4} /{ }^{\circ} \mathrm{C}$, independent of the compositions, and are very close to other organic polymers. ${ }^{1}$

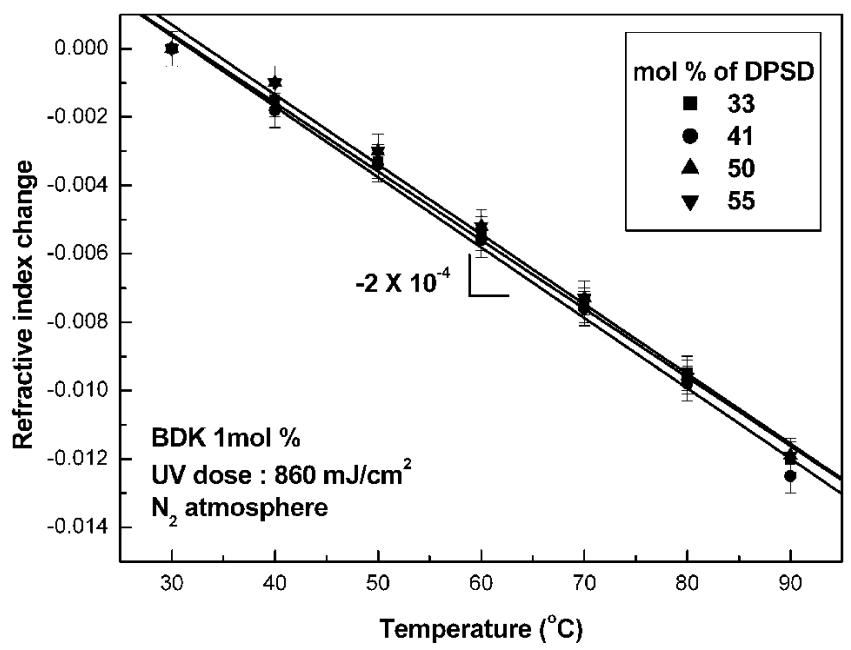

FIG. 8. Refractive index change in the methacryl HYBRIMER films with different composition as a function of temperature.
According to the Prod'homme theory, the $\mathrm{d} n / \mathrm{d} T$ can be described $\mathrm{as}^{20}$

$$
\frac{\mathrm{d} n}{\mathrm{~d} T}=f(n)(\Phi-\beta)
$$

where $f(n)=\left(n^{2}-1\right)\left(n^{2}+2\right) / 6 n, \Phi$ is the temperature coefficient of electronic polarizability, and $\beta$ is the thermal expansion coefficient.

When the $\Phi$ is dominant, the refractive index becomes positive and increases with temperature. This is a characteristic of inorganic materials. When $\beta$ dominates, on the other hand, $\mathrm{d} n / \mathrm{d} T$ becomes negative, and it decreases with the increase in temperature. This is a characteristic of organic polymers. The $\mathrm{d} n / \mathrm{d} T$ 's of most organic polymers are actually negative and are at $10^{-4} /{ }^{\circ} \mathrm{C}$, since their $\beta s$ are at $10^{-4} /{ }^{\circ} \mathrm{C}$. It can, therefore, be expected that the thermo-optic characteristic of the methacryl HYBRIMER film originated mainly from a thermal expansion behavior, similar to that of organic polymers.

It has already been reported in previous works that both the formation of siloxane bond and organic networks in other HYBRIMER films can positively increase the value of the $\mathrm{d} n / \mathrm{d} T$ since their thermal expansion

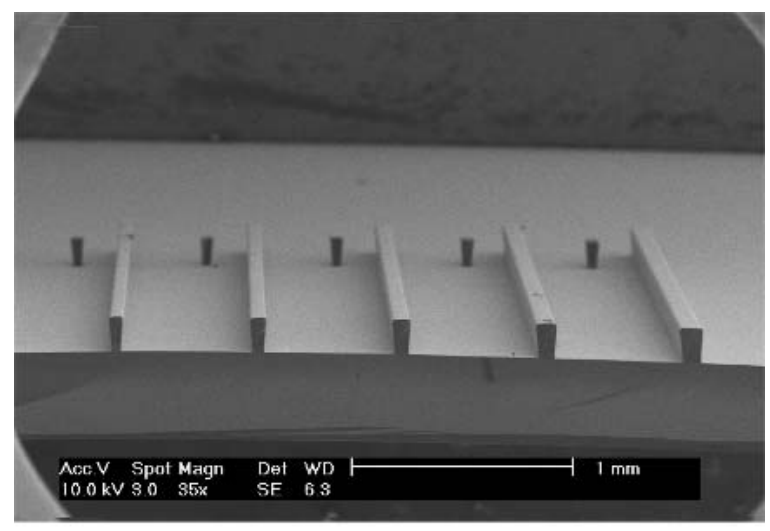

(a)

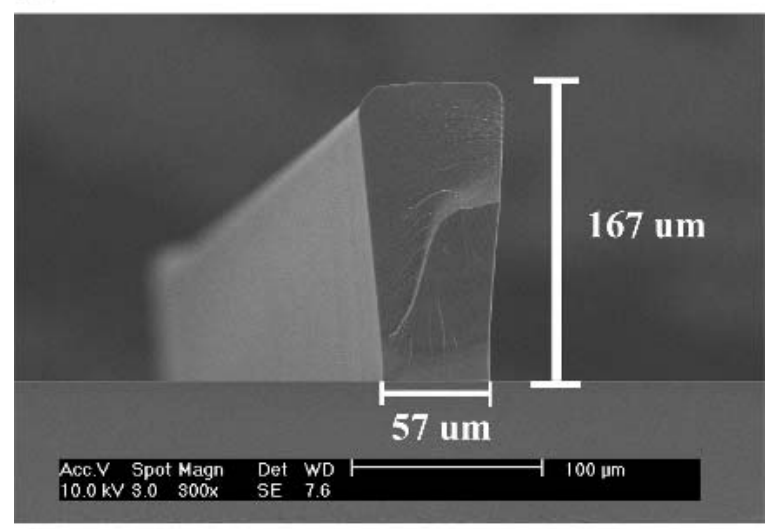

(b)

FIG. 9. SEM images of the photo-patterns of the methacryl HYBRIMER films (a) using a photomask with 60-100 $\mu \mathrm{m}$ slit pattern and a 10- $\mu \mathrm{m}$ width difference, and (b) obtained with $60 \mu \mathrm{m}$ slit in (a). 
coefficients have been reduced by cross-linking. ${ }^{7,18,21}$ As the DPSD contents increase, the formation of the siloxane bond is promoted due to the further condensation reaction. The degree of polymerization of the methacryl group, on the other hand, is reduced by the steric effect, as shown in Fig. 6. As a result, these two effects are mutually compensated in thermo-optic behavior of the methacryl HYBRIMER films, and the value of the $\mathrm{d} n / \mathrm{d} T$ can remain constant in all the compositions.

\section{E. Photo-patterning behavior of thick films}

The fabrication of a photo-pattern for optical applications is generally carried out through a multi-step process, which includes photo-resist deposition, UV exposure, development, and wet or dry etching. ${ }^{1}$ The methacryl HYBRIMER can, however, be directly photopatternable without the photo-resist deposition step, since the unpolymerized resin in the unexposed area is removed by developing. This photo-patterning merit makes it easy for the methacryl HYBRIMER to develop planar optical devices on chips.

The photo-patterning of thick HYBRIMER films with 50-mol\% DPSD contents was performed using a photomask and a metal spacer. The resin was spun on the $\mathrm{Si}(100)$ wafer at $200 \mathrm{rpm}$, and the metal spacer $(200-\mu \mathrm{m}$ thickness) was placed on the Si wafer to prevent direct contact between the film and the photo-mask. The photomask was then put on the metal spacer. The film was illuminated with a UV lamp through the photo-mask at UV doses of $860 \mathrm{~mJ} / \mathrm{cm}^{2}$. The sample was developed for $30 \mathrm{~s}$ in an n-propylacetate/isopropyl alcohol mixture (volume ratio 1:1) after UV exposure and subsequently rinsed with isopropyl alcohol.

Figure 9(a) exhibits the SEM images of the photopatterns of the methacryl HYBRIMER film using a photo-mask with $60-100 \mu \mathrm{m}$ slit pattern and a $10-\mu \mathrm{m}$ width difference. Figure 9(b) shows the pattern with the $60-\mu \mathrm{m}$ slit in Fig. 9(a). Even in films up to $170 \mu \mathrm{m}$ thick, a high aspect ratio (3:1) pattern can be obtained. This reveals that the thick photo-curable methacryl HYBRIMER can be used in the fabrication of various optical devices and Micro Electro Mechanical System.

\section{CONCLUSION}

Methacryl-oligosiloxane nano hybrid (methacryl HYBRIMER) films were fabricated from MPTS and DPSD, and the effects of compositional modification on their optical properties were verified. The viscosity of the methacryl-oligosiloxane nano hybrid resin was altered at $10^{2}$ intervals by the compositional modification but without any drastic changes in the reaction parameters, and thickness controllable (from 11 to $150 \mu \mathrm{m}$ ) and uniform (less than $1 \mathrm{~nm}$ rms roughness) coating films were obtained through a single-coating step. The degree of photo-polymerization of the resin decreased with the increase in DPSD contents, as a result of the steric effect. The refractive indices of the films increased nonlinearly from 1.506 to 1.543 with the increase in DPSD contents, due to the high electronic polarizability of the phenyl group and the densification induced by photopolymerization. On the other hand, the thermo-optic coefficients remained constant $\left(-2 \times 10^{-4} /{ }^{\circ} \mathrm{C}\right)$, independent of composition, due to the compensation between the degree of condensation and photo-polymerization. And also, we demonstrated a thick $(170 \mu \mathrm{m})$ photo-pattern with high aspect ratio (3:1). The methacryl-oligosiloxane nano hybrid materials can be a promising candidate for the optical application owing to their wide tunability of optical parameters through the simple compositional modification.

\section{ACKNOWLEDGMENT}

This work was supported by the Sol-Gel Innovation Project (SOLIP) of the Ministry of Commerce, Industry, and Energy of Korea.

\section{REFERENCES}

1. L. Eldada and L.W. Shacklette: Advances in polymer integrated optics. IEEE J. Sel. Top. Quantum Electron. 6, 54 (2000).

2. B.L. Booth: Low loss channel waveguides in polymers. J. Lightwave Technol. 7, 1445 (1989).

3. J. Ballato, R.E. Riman, and E. Snitzer: Sol-gel synthesis of fluoride optical materials for planar integrated photonic applications. J. Non-Cryst. Solids 213, 126 (1997).

4. M. Yoshida and P. Prasad: Fabrication of channel waveguides from sol-gel-processed polyvinylpyrrolidone/ $\mathrm{SiO}_{2}$ composite materials. Appl. Opt. 35, 1500 (1996).

5. U. Schubert, N. Husing, and A. Lorenz: Hybrid inorganic-organic materials by sol-gel processing of organofunctional metal alkoxides. Chem. Mater. 7, 2010 (1995).

6. T. Watanabe, N. Ooba, S. Hayashida, T. Kurihara, and S. Imamura: Polymeric optical waveguide circuits formed using silicone resin. J. Lightwave Technol. 16, 1049 (1998).

7. E.S. Kang, J.U. Park, and B.S. Bae: Effect of organic modifiers on the thermo-optic characteristics of inorganic-organic hybrid material films. J. Mater. Res. 18, 1889 (2003).

8. O.H. Park, S.Y. Seo, J.I. Jung, J.Y. Bae, and B.S. Bae: Photoluminescence of mesoporous silica films impregnated with an erbium complex. J. Mater. Res. 18, 1039 (2003).

9. O.H. Park, J.I. Jung, and B.S. Bae: Photoinduced condensation of sol-gel hybrid glass films doped with benzildimethylketal. J. Mater. Res. 16, 2143 (2001).

10. O.H. Park, S.J. Kim, and B.S. Bae: Photochemical reactions in fluorinated sol-gel hybrid materials doped with a photolocking agent for direct micropatterning. J. Mater. Chem. 14, 1749 (2004).

11. J.H. Jang, D.J. Kang, and B.S. Bae: Large photoinduced densification in organically modified germanosilicate glasses. J. Am. Cerm. Soc. 87, 155 (2004).

12. W.S. Kim, R. Houbertz, T.H. Lee, and B.S. Bae: Effect of photoinitiator on photopolymerization of inorganic-organic hybrid polymers (ORMOCER). J. Polym. Sci., Part B: Polym. Phys. 42, 1979 (2004). 
13. R. Buestrich, F. Kahlenberg, M. Popall, P. Dannberg, R. Müller-Fieldler and O. Rösch: ORMOCER ${ }^{\circledR}$ S for optical interconnection technology. J. Sol-Gel Sci. Technol. 20, 181 (2001).

14. R. Houbertz, J. Schulz, L. Fröhlich, G. Domann, and M. Popall: Inorganic-organic hybrid materials for polymer electronic applications, in Flexible Electronic-Materials and Device Technol$o g y$, edited by N. Fruehauf, B.R. Chalamala, B.E. Gnade, and J. Jang (Mater. Res. Soc. Symp. Proc. 769, Warrendale, PA, 2003), p. H7.4.1.

15. U. Streppel, P. Dannberg, C. Wächter, A. Bräuer, L. Fröhlich, R. Houbertz, and M. Popall: New wafer-scale fabrication method for stacked optical waveguide interconnects and 3D micro-optic structures using photoresponsive (inorganic-organic hybrid) polymers. Opt. Mater. 21, 475 (2002).

16. R. Buestrich, F. Kahlenberg, M. Popall, A. Martin, and O. Rösch: Lo Si-OH ORMOCERS $®$ for dielectrical and optical interconnection technology, In Organic/Inorganic Hybrid Materials—2000, edited by R.M. Laine, C. Sanchez, C.J. Brinker, and E. Giannelis (Mater. Res. Soc. Symp. Proc. 628, Warrendale, PA, 2000), p. CC9.8.1.

17. Y.J. Eo, T.H. Lee, S.Y. Kim, J.K. Kang, Y.S. Han, and B.S. Bae: Synthesis and molecular structure analysis of nano-sized methacryl-grafted polysiloxane resin for fabrication of nano hybrid materials. J. Polym. Sci., Part B: Polym. Phys. (accepted).

18. E.S. Kang, T.H. Lee, and B.S. Bae: Measurement of thermo-optic coefficients in sol-gel derived inorganic-organic hybrid material films. Appl. Phys. Lett. 81, 1438 (2002).

19. M.P. Stevens: Polymer Chemistry, 3rd ed. (Oxford University Press, Oxford and New York, 1999), p. 65.

20. L. Prod'homme: A new approach to the thermal change in the refractive index of glasses. Phy. Chem. Glasses 4, 119 (1960).

21. J. Zahi, L. Qiu, J. Zhou, Y. Zaho, Y. Shen, Q. Ling, and M. Yang: Study on the thermal properties of doped PMMA systems. Adv. Mater. Opt. Electron. 10, 3 (2000). 